\title{
Time series analysis of dengue incidence in Guadeloupe, French West Indies: Forecasting models using climate variables as predictors
}

\author{
Myriam Gharbi 1,6* Philippe Quenel ${ }^{2}$, Joël Gustave3 ${ }^{3}$, Sylvie Cassadou², Guy La Ruche ${ }^{4}$, Laurent Girdary ${ }^{5,6}$ and
} Laurence Marrama ${ }^{6}$

\begin{abstract}
Background: During the last decades, dengue viruses have spread throughout the Americas region, with an increase in the number of severe forms of dengue. The surveillance system in Guadeloupe (French West Indies) is currently operational for the detection of early outbreaks of dengue. The goal of the study was to improve this surveillance system by assessing a modelling tool to predict the occurrence of dengue epidemics few months ahead and thus to help an efficient dengue control.

Methods: The Box-Jenkins approach allowed us to fit a Seasonal Autoregressive Integrated Moving Average (SARIMA) model of dengue incidence from 2000 to 2006 using clinical suspected cases. Then, this model was used for calculating dengue incidence for the year 2007 compared with observed data, using three different approaches: 1 year-ahead, 3 months-ahead and 1 month-ahead. Finally, we assessed the impact of meteorological variables (rainfall, temperature and relative humidity) on the prediction of dengue incidence and outbreaks, incorporating them in the model fitting the best.
\end{abstract}

Results: The 3 months-ahead approach was the most appropriate for an effective and operational public health response, and the most accurate (Root Mean Square Error, RMSE $=0.85$ ). Relative humidity at lag-7 weeks, minimum temperature at lag-5 weeks and average temperature at lag-11 weeks were variables the most positively correlated to dengue incidence in Guadeloupe, meanwhile rainfall was not. The predictive power of SARIMA models was enhanced by the inclusion of climatic variables as external regressors to forecast the year 2007. Temperature significantly affected the model for better dengue incidence forecasting ( $p$-value $=0.03$ for minimum temperature lag-5, $p$-value $=0.02$ for average temperature lag-11) but not humidity. Minimum temperature at lag-5 weeks was the best climatic variable for predicting dengue outbreaks (RMSE $=0.72$ ).

Conclusion: Temperature improves dengue outbreaks forecasts better than humidity and rainfall. SARIMA models using climatic data as independent variables could be easily incorporated into an early ( 3 months-ahead) and reliably monitoring system of dengue outbreaks. This approach which is practicable for a surveillance system has public health implications in helping the prediction of dengue epidemic and therefore the timely appropriate and efficient implementation of prevention activities.

Keywords: Dengue fever, Time series analysis, SARIMA models, Forecasting, Climate, Guadeloupe, America region

\footnotetext{
* Correspondence: myriam.gharbi@wwarn.org

${ }^{1}$ Ecole Pasteur-Cnam de Santé Publique, Paris, France

Full list of author information is available at the end of the article
} 


\section{Background}

Dengue is a human arbovirus disease transmitted by the female mosquito of the genus Aedes, mainly Aedes aegypti and Ae. albopictus [1]. Dengue, the most frequent arthropod-borne viral disease, is prevalent in tropical and subtropical regions. Two major clinical forms of dengue illness involve the mild form of dengue fever and severe form mostly characterized by plasma leakage with or without haemorrhage [2].

Two-fifths of the world population (about 2.5 billion people) is at risk of dengue infection. The prevalence of this disease has grown dramatically in the recent decades. Between 50 and 100 million people are infected each year worldwide and more than 500,000 are hospitalized [3]. The average annual incidence was multiplied by thirty in the last fifty years. Incidence of dengue haemorrhagic fever (DHF) is increasing in many tropical regions inducing 20,000 deaths per year, mostly among children under 15 years [4].

In the Caribbean and Latin America countries, the reintroduction and dissemination of Ae aegypti were observed in the 1970s, after the reduction of vector control interventions initiated in the 1960s. Since that time, regular outbreaks occured with a cycle of 3-5 years, combined with an increase in severe forms, particularly DHF [5]. In some areas, as the French Overseas Territories of the Americas (FOTAs: French West Indies, Martinique and Guadeloupe, and French Guyana), the epidemiology of dengue is moving from an endemo-epidemic situation towards a hyper-endemic situation [6]. Over the decade 1997-2007, the FOTAs had four major epidemics (1997, 2001, 2005 and 2007) each linked to the circulation of one or two predominant serotypes. These outbreaks usually last 4-6 months and may affect up to $5 \%$ of the population. The epidemiological dynamics observed over this decade in the FOTAs raises fears of a move towards a situation comparable to that currently in South East Asia and dengue could become one of the leading causes of hospitalization, especially for children. During the last two epidemics of dengue in Guadeloupe (400,500 inhabitants in 2007), the number of clinical cases that led to a medical consultation were respectively 11,500 in 2005 ( $0.4 \%$ of severe cases; serotype 4 was predominant) and 19,000 in 2007 (0.8\% of severe cases; serotype 2 predominant).

Dengue is endemic in all surrounding countries with the four serotypes circulating in the region within a period of ten years. Countries or territories with the highest number of reported dengue cases were Puerto Rico, the Dominican Republic, Martinique, Trinidad and Tobago and French Guiana.

Population movement is an important factor in the virus dissemination. It contributes to carry new virus strains but it also participates to introduce non immune subjects in an endemic area. Dengue outbreaks may occur when a high proportion of naïve subjects are concentrated in the same area. As the social and economic impacts are worsening $[7,8]$ and outbreaks are increasing, it becomes urgent to reinforce an integrated management for the surveillance, control and prevention of dengue [9]. One key aspect of this strategy is the ability to predict the occurrence of dengue outbreaks.

An early warning of dengue outbreaks could improve the efficiency of vector control campaigns and help to target prevention actions. Such early interventions could delay or spread out the epidemic, thus reducing its impact on health system. Health facilities could adapt their response in terms of availability of beds and mobilization of human and material resources [10]. Dengue morbidity and mortality would be minimized through earlier and more appropriate public health response.

Many complex mathematical models have been developed to predict the occurrence, dynamics and magnitude of outbreaks using a combined environmental and biological approach. Various parameters have been used [11,12] such as climatic data $[13,14]$, vector characteristics $[15,16]$, availability of breeding sites [14], viral serotypes circulating, immune status of host populations [13] or demographics data [17]. Meteorological conditions are considered as some of the most important factors of dengue in outbreaks occurrence [18]. Many studies have highlighted the relationship between climate and dengue transmission. The increase of temperature has been found associated with dengue in Thailand [19,20], Indonesia [21], [22] and [23], Singapore [24], Mexico [25] and Puerto Rico [26]. Elevated humidity with high mosquito density increased the transmission rate of dengue fever infection in southern Taiwan [27]. Meanwhile, a large amount of rainfall has been linked to dengue fever in Indonesia [23], Trinidad [28], Venezuela [29], Barbados [30] and Thailand [20].

These models, requiring important human and logistical resources for data collection and implementation, are difficult to be used for a continuous surveillance system and for early detection, particularly in developing countries. Therefore, it is important to develop a surveillance system based on a model that includes data as explanatory variables [31], respecting the following steps:

- To develop a model that correctly identifies and quantifies the relationship between dengue and climatic variables;

- To reduce the model to its most parsimonious form so that human and material resources could be focused on the collection of essential data;

- To ensure that the surveillance system is ongoing and sustainable and able to record cases at regular intervals (weekly or monthly). 
The surveillance system in Guadeloupe is currently operational for the detection of early outbreaks of dengue. The aim of the study is to improve this surveillance system by assessing a modelling tool including meteorological data to predict the occurrence of dengue epidemics few weeks or months ahead (duration consistent with greater anticipation of outbreaks). This predicable model would be used to help an efficient dengue control.

We propose to develop Seasonal Autoregressive Integrated Moving Average (SARIMA) models using time series analysis of dengue incidence. Such models are particularly interesting when there are time dependences between each observation [32]. The assumption that each observation is correlated to previous ones makes it possible to model a temporal structure, with more reliable predictions, especially for seasonal infections [33-36], than those obtained by other statistical methods. SARIMA models have been successfully used in epidemiology to predict the evolution of infectious diseases, such as malaria and hepatitis A [35], deaths due to influenza [37] and pneumonia [33]. Moreover, these models allow the integration of external factors, such as climatic variables, that may increase their predictive power [38,39].

A prediction approach has been developed in this study using data from Guadeloupe. It could be implemented regionally or internationally, if it is evaluated and validated in other part of Caribbean and Latin America (beginning with others FOTAs).

\section{Methods}

\section{Settings}

Guadeloupe, with a surface of $1,434 \mathrm{~km}^{2}$, is an island located in the Caribbean, between the Tropic of Cancer and the Equator (latitude $15^{\circ} 57^{\prime}-16^{\circ} 31^{\prime}$ North and longitude $61^{\circ} 10^{\prime}-61^{\circ} 48^{\prime}$ West). The climate is tropical with two distinct seasons: a dry season from January to June, characterized by relatively low rainfall and a wet season from July to December.

\section{Data Collection}

This study covers the period from 2000 to 2007 for dengue incidence and meteorological data. During the study period, stable dengue control programs were implemented each year in Guadeloupe.

The sentinel network was set up from 1983 by local health authorities in association with Institut Pasteur in Guadeloupe. Until 2004, dengue-like syndrome reporting was linked to laboratory confirmation done by Institut Pasteur [40]. Then the monitoring of dengue-like syndromes was going on with sentinel physicians but was separated from confirmed case surveillance, as more and more laboratories were involved in diagnostic confirmation. Nowadays, epidemiologic surveillance system of dengue in Guadeloupe is based on 3 main indicators collected independently: (1) weekly number of dengue-like syndromes (suspected dengue cases) collected from sentinel general practitioners (GPs) for early detection and measurement of magnitude of epidemics; (2) weekly number of confirmed cases of dengue from all laboratories (hospital or not) for confirmation of virus circulation; (3) monthly number of confirmed hospitalized cases from hospital wards for measurement of epidemics severity. Thus, laboratory-confirmed cases, which is a less efficient indicator to monitor the dynamics of an epidemic, and syndromes dengue-like counted for surveillance are not directly linked. In this study, the dengue incidence was calculated from the weekly number of dengue-like syndromes and data from the Institut Pasteur were only used for validation purpose of this incidence.

The number of sentinel GPs, as well as the representativeness of the network in terms of GP's activity, has progressively improved to reach a quite good representativeness (it represents $12 \%$ of all GPs in Guadeloupe in 2008 ) with a high weekly participation rate $(85 \%$ on average in 2008). Nevertheless, it could be qualitatively improved for a better adequacy with the geographical distribution of the population [41]. Dengue cases reported by practitioners are mainly non-severe; severe cases treated in hospitals are not included but represent less than $1 \%$ of dengue cases.

A suspected dengue case is defined as a patient with less than seven days of fever $\left(\geq 38.5^{\circ} \mathrm{C}\right)$ without evidence of other cause of infection and with at least one symptom of pain (headache, retro-orbital pain, myalgia, arthralgia, back pain).

Each week, the total number of dengue cases collected is extrapolated to the whole island using for adjustment the ratio "medical activity of sentinel GPs present during the week"/"medical activity of all the GPs in Guadeloupe". Weekly incidence rates were calculated using demographic data for Guadeloupe from the National Institute of Statistics and Economic studies. Missing data were replaced using a simple exponential smoothing method. The time series were adjusted by adding the constant of one to the data. This procedure did not change the temporal structure of the series and allowed the use of a logarithmic transformation.

The meteorological station of Raizet (the international airport of Guadeloupe) records meteorological variables every day: cumulative rainfall $(\mathrm{mm})$, relative humidity (\%), minimum, maximum and average temperature $\left({ }^{\circ} \mathrm{C}\right)$. The availability of these data explains the use of these climatic variables in our study. These data, aggregated on a weekly basis, cover the study period without any missing values. 


\section{Processing and data analysis}

A SARIMA model was adjusted to the data of dengue incidence from 2000 to 2006, using the Box and Jenkins approach developed in $1974[32,34,35,42,43]$. This model allowed predicting dengue incidence for the year 2007.

Box-Jenkins method is a four-step process:

Firstly, the variance was stabilized using an appropriate transformation (logarithmic, square root or inverse transformation), based on a mean-range plot analysis as a relevant decision tool. The mean was also stabilized by differencing according to the profile of the original time series. A seasonal component was removed by a seasonal differencing: $\mathrm{Zt}$ - Zt-s $(\mathrm{Zt}=$ values of the time series at time $\mathrm{t}$ and $\mathrm{Zt}-\mathrm{s}=$ values of the time series at time $\mathrm{t}-\mathrm{s}$ with $s$ corresponding to the seasonality) and the trend was removed by a regular differencing: $\mathrm{Zt}-\mathrm{Zt}-1$ (Zt-1 = values of the time series at time $t-1$ week). The seasonality as well as the trend are highlighted by plotting the original time series. The presence of these two components determine the choice of a SARIMA model equation: $y_{t}=\frac{\Theta_{q}(B) \Theta_{Q}\left(B^{S}\right) a_{t} \Phi_{P}\left(B^{S}\right)}{\Phi_{P}\left(B^{S}\right) \Phi_{P}(B)(1-B)^{d}\left(1-B^{S}\right)^{D}}$ where $\Phi_{P}\left(B^{S}\right)$ is the seasonal autoregressive (AR) operator, $\Phi_{P}(B)$ AR the operator, $\Theta_{q}(B)$ the moving average (MA) operator, $\Theta_{Q}\left(B^{S}\right)$ the seasonal MA operator, $(1-B)^{d}$ and $\left(1-B^{S}\right)^{\mathrm{D}}$ the ordinary and seasonal difference components, $a_{t}$ the white noise, and $y_{t}$ the dependant variable.

The choice of the order of integration $(D, d)$ parameters is based on the plot of integrated time series and based on comparison of their standard deviation; the series having the more stabilized mean is selected.

Secondly, the temporal structure of the series, i.e. order of seasonal and non seasonal AR (P, p), MA (Q, q) parameters, were determined. Several tools are available:

- To identify the order of MA and AR parameters, the structure of temporal dependence of stationary time series is assessed respectively, by the analysis of autocorrelation (ACF) and partial autocorrelation (PACF) functions.

- To select the model, with fewer parameters that fits the data best, the Akaïke Information Criterion (AIC) is used.

- To validate the final model, its residuals are analyzed by the Ljung-Box test. Residuals must be equivalent to white noise.

Thirdly, model parameters were estimated by the maximum likelihood method.

Finally, predicted data for 2007 were compared with observed data in order to validate the model. The average error was calculated using the RMSE (Root Mean Square
Error) equals to: $R M S E=\left[\frac{\sum_{t=1}^{N}\left(Y_{t}-\hat{Y}_{t}\right)^{2}}{N}\right]^{1 / 2}$ with $Y_{t}$ the observed value and $\hat{Y}_{t}$ the predicted value at $t$ time and $\mathrm{N}$ the number of observations.

The predictions and their 95\% confidence intervals were estimated for 2007 using the best model fitting the data for 2000-2006. Three different methods were compared; the first consisted to predict 2007 data with a one year (52 weeks) lag; the second and third methods were iterative approaches. They consisted to predict dengue incidence, with three (13 weeks) and one month (4 weeks) lags, respectively. After the prediction of the first period, the observed data for this period were included in the database in order to update the model and estimate the predictions for the second period. The same were used to complete the predictions for the whole year 2007.

Once the univariate model was selected, the multivariate models including external regressors could be elaborated. Meteorological variables were used to improve the predictive power of the model. In order to include these explanatory variables, cross-correlation graphics (Pearson test) between data of dengue incidence and climatic variables for a 16 weeks period were performed.

As the use of cross-correlations on original time series is not recommended, they were performed on the residuals of the models obtained by applying a SARIMA model to each series [44]. Climatic variables significantly associated to dengue incidence were tested as predictors in a multivariate SARIMA model. The model equation is $y_{t}=\frac{\Theta_{q}(B) \Theta_{Q}\left(B^{S}\right) a_{t} \Phi_{P}\left(B^{S}\right)}{\Phi_{P}\left(B^{S}\right) \Phi_{P}(B)(1-B)^{d}\left(1-B^{S}\right)^{D}}+X$, where $X$ is the external variable.

This model was able to give predictions with a lag of one, three or twelve months, depending on the approach selected during the univariate process. The predictive power of the models was estimated with the RMSE (a significant decrease of RMSE denotes an improvement of the model) and the Wilcoxon signed-ranks test which was used for significance assessment.

The statistical software $\mathrm{R}$ (version 2.9.0) and Statgraphics were used for all analyses. Statgraphics allowed a first approach of the problem. $\mathrm{R}$ had confirmed all items found on Statgraphics. The graphics were performed with $\mathrm{R}$.

\section{Results}

The plot of the observed dengue incidence (Figure 1) showed three major outbreaks in Guadeloupe (late 2001 early 2002, mid 2005 - early 2006, mid 2007 - late 2007). The bivariate analysis between crude climatic variables and dengue incidence shows that the three major 
A

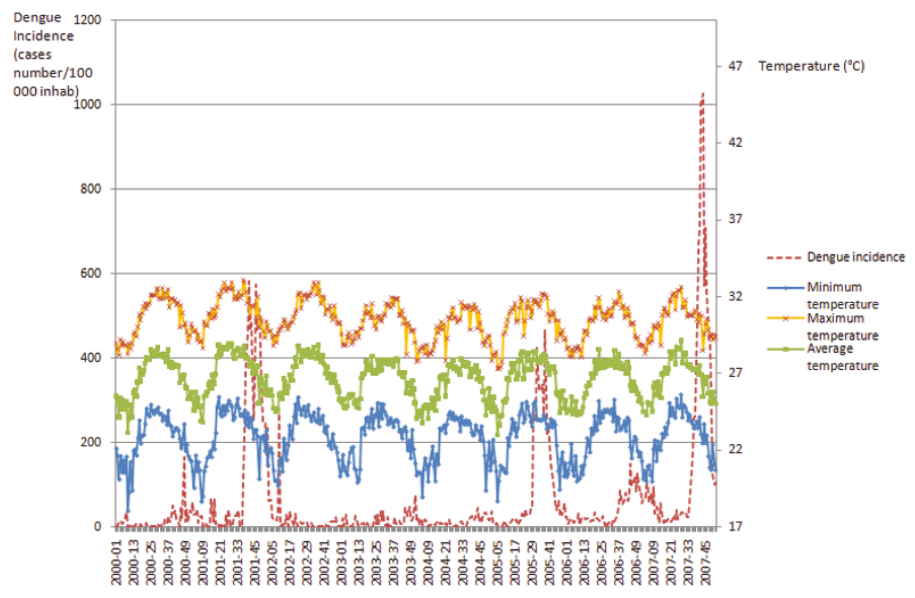

B

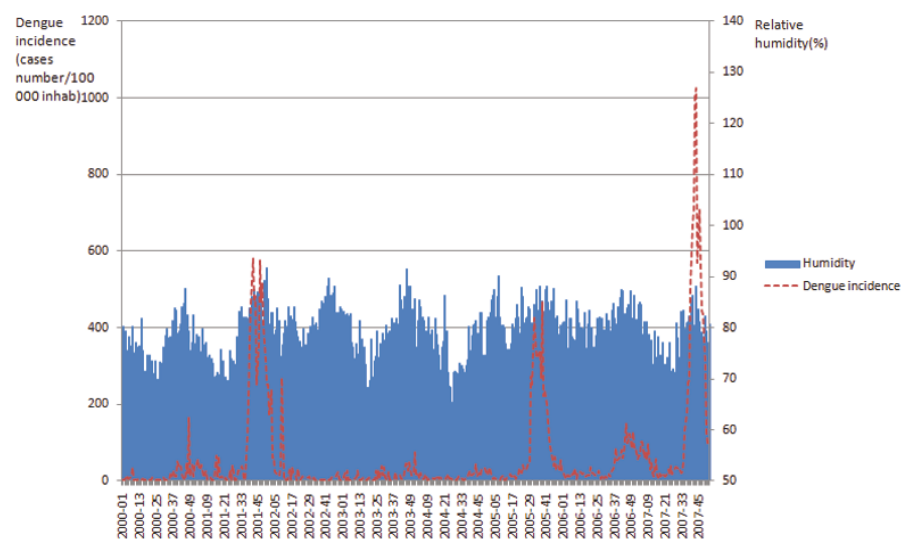

C

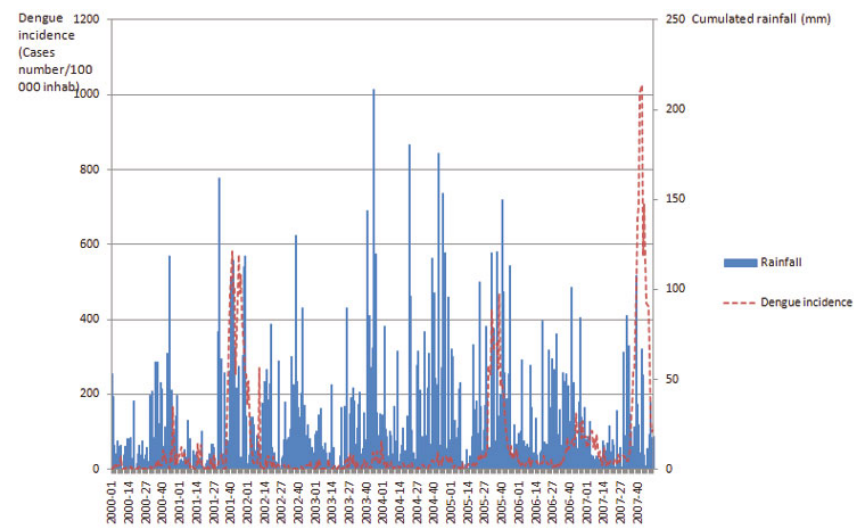

Figure 1 Dashed red line: Weekly incidence rates of dengue (per 100,000) in Guadeloupe from January 2000 to December 2007 compared to crude meteorological variables for the same period: A) minimum temperature (blue diamond), maximum temperature (yellow cross) and average temperature (green square); B) relative humidity (blue area); C) weekly cumulated rainfall (blue solid line). 
outbreaks were correlated to a slight decrease of temperature (Figure 1A) and to an increase in relative humidity (Figure 1B). Dengue incidence was not clearly correlated to weekly cumulated rainfall (Figure 1C). An annual seasonality is identified for all these meteorological variables.

In the first step of the dengue time series analysis, it has been necessary to stabilize the variance of the series by natural logarithm transformation. This transformation had the lowest dispersion. To stabilize the mean, seasonal and regular differencing were applied. The plots of ACF and PACF (Figures 2A and 2B) showed the temporal dependence of the dengue incidence and confirmed the need to use a SARIMA model with seasonal (P, D, Q) and non-seasonal (p, d, q) parameters. After differencing, a significant cut-off at one week-lag and another at lag-52 weeks were observed on the plot ACF (Figure 2C). These two cut-offs were less marked on the plot PACF (Figure 2D) and evolve more gradually over the time, compared to the plot ACF. Therefore, the following univariate multiplicative SARIMA $(0,1,1)$ $(0,1,1)_{52}$ model was the best to fit the dengue incidence $($ AIC $=951$, Table 1$)$. The analyses of residuals on ACF and PACF plots (Figure 2E and 2F) assessed the absence of persistent temporal correlation. The Ljung-Box test confirmed that the residuals of time series were statistically not dependent ( $\mathrm{p}$-value $>0.05)$. The selected
Table 1 Coefficients, standard errors, $t$ statistic and P-value of the parameters of the $\operatorname{SARIMA}(0,1,1)(0,1,1)_{52}$ model estimated by maximun likelihood

\begin{tabular}{ccccc}
\hline Parameters & Coefficients & $\begin{array}{c}\text { Standard } \\
\text { error }\end{array}$ & T statistic & P-value \\
\hline Non seasonal MA (q) & -0.6092 & 0.0481 & -12.66 & $9.56 \mathrm{e}-37^{*}$ \\
\hline Seasonal MA (Q) & -0.8858 & 0.1875 & -4.72 & $2.32 \mathrm{e}-06^{*}$ \\
\hline
\end{tabular}

MA: moving average, *:p-value $<0.05$ significant.

SARIMA model fitted observed data from 2000 to 2006 $($ RMSE $=0.914)$. For 2007, the 3 methods were compared (Figure 3). The 4 weeks-step approach showed the smallest difference between observed and predicted values $($ RMSE $=0.76)$ when compared to the 52 weeksstep approach $(\mathrm{RMSE}=0.98)$ and to the 13 weeks-step approach (RMSE $=0.85)$. However, the difference between residuals predicted 13 weeks-ahead and those predicted 4 weeks-ahead was not statistically significant (Wilcoxon signed-ranks, p-value $=0.48$ ). The predictions for the 3 following months were the best compromise for helping the health authorities to take measures to mitigate transmission, morbidity and mortality.

To include climatic variables (time series) as external variables in the univariate model, a SARIMA model was applied to all time series (Table 2). Cumulative rainfall and relative humidity variables were $\log$ transformed. A

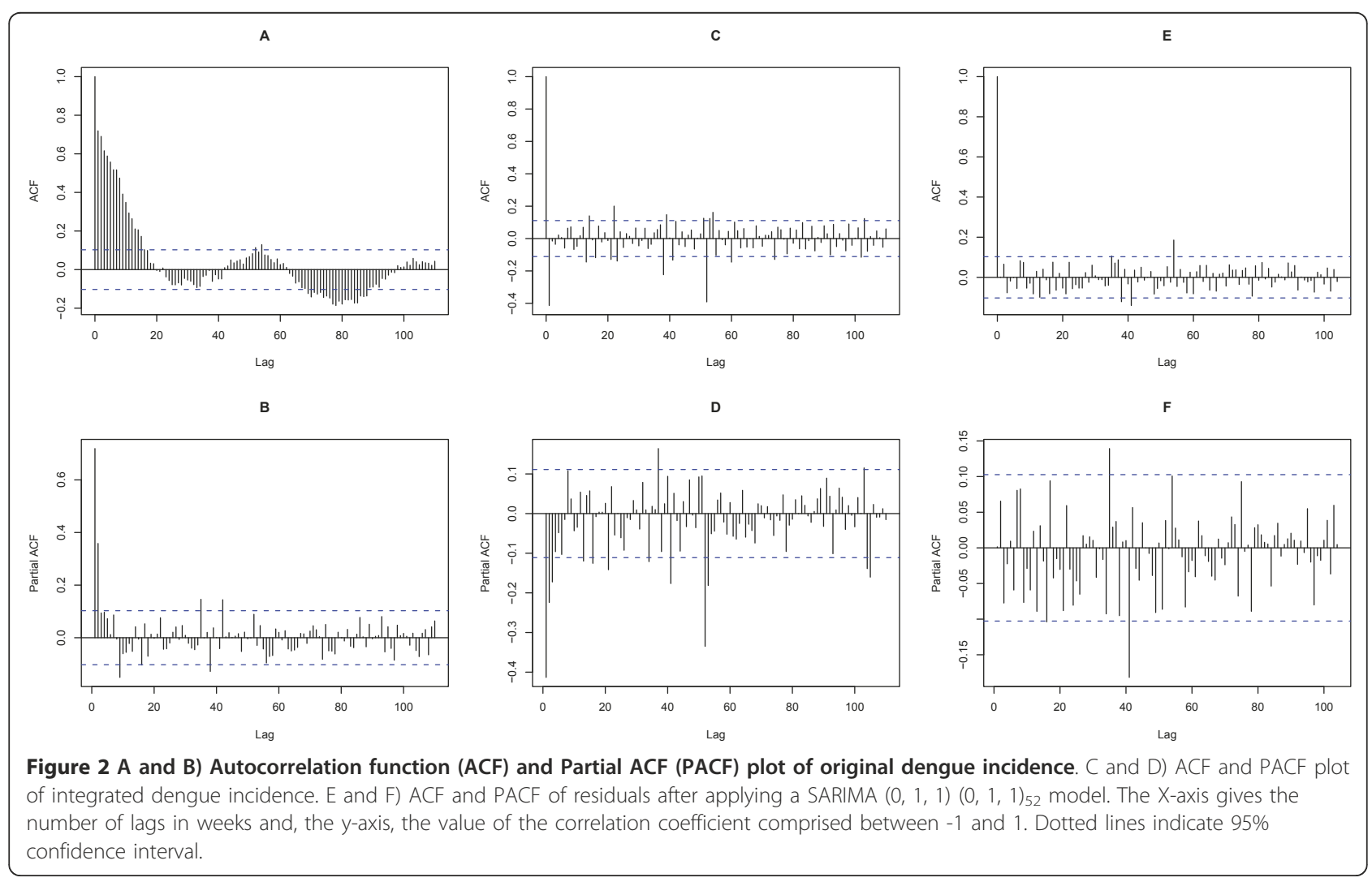




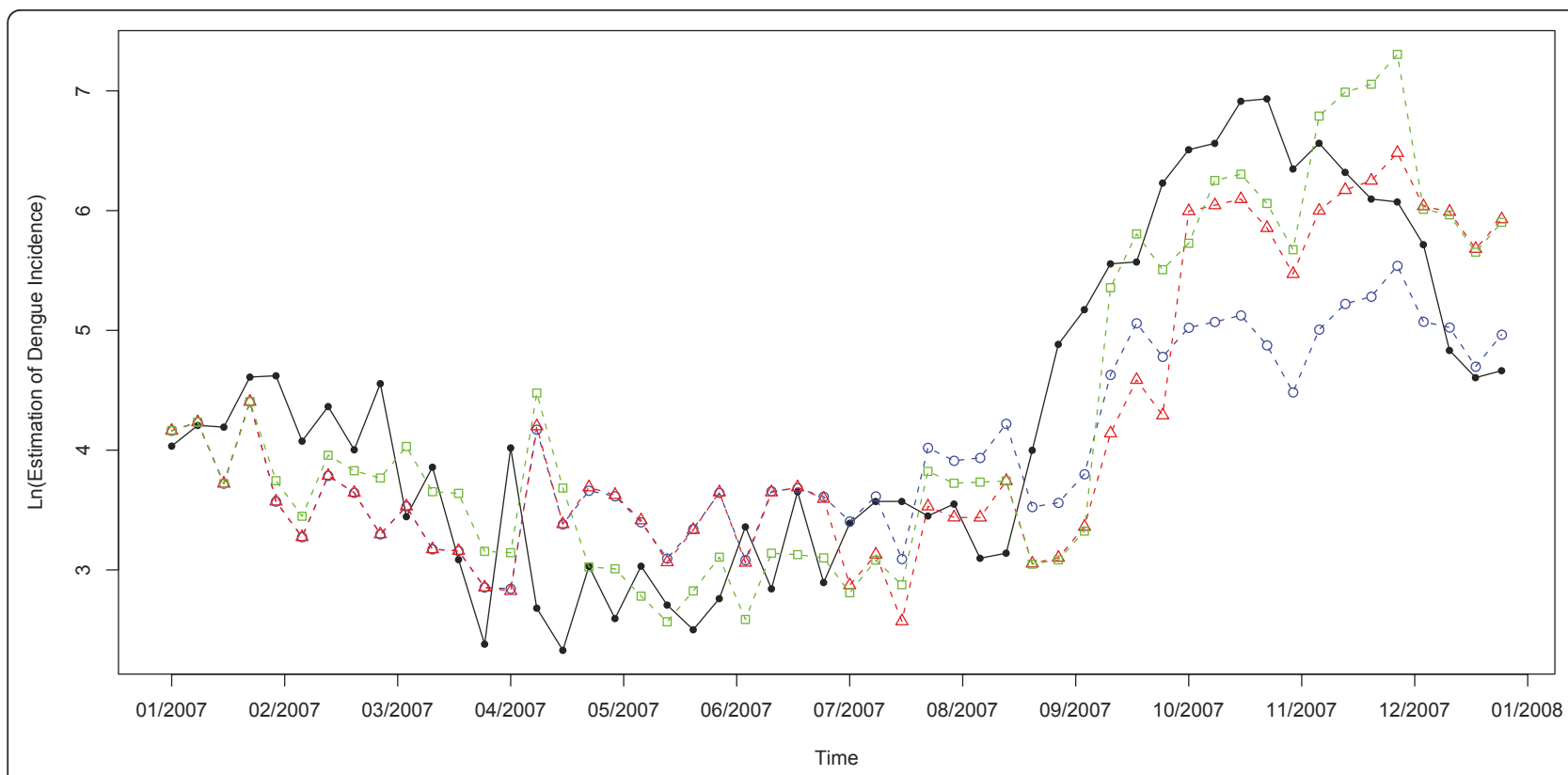

Figure 3 Natural logarithm of dengue incidence in Guadeloupe for 2007. Solid line (filled circle): observed values during the period. Dashed lines: univariate SARIMA $(0,1,1) \times(0,1,1) 52$ model; blue (circle): 52 weeks ahead values; red (triangle): 13 weeks ahead values; green (square): 4 weeks ahead values.

regular differencing was applied for all variables except for minimum temperature. A seasonal differencing was also applied for all variables except for relative humidity. The residuals were kept for the multivariate analyses.

Then, correlations between residuals of dengue incidence and those of meteorological variables over a range of 16 weeks-lags were analyzed. Climatic variables identified as the most correlated to dengue incidence were included one by one, because of their strong interrelationship, to test their influence on the model. The following explanatory variables were the most positively correlated with dengue incidence: lag-5 minimum temperature (Pearson correlation: $\mathrm{r}=0.118, \mathrm{p}$-value $=$ $0.025)$, lag- 11 average temperature $(\mathrm{r}=0.141, \mathrm{p}$-value $=$ $0.007)$ and lag-7 relative humidity $(\mathrm{r}=0.107, \mathrm{p}$-value $=$ 0.04), (Figure 4). Meanwhile, rainfall was not correlated with dengue incidence over a range of 16 weeks-lags ( $r$ $<0.06, \mathrm{p}$-value $=$ non significant).
SARIMA models $(0,1,1)(0,1,1)_{52}$ including external independent variables, either the lag-5 minimum temperature or the lag-11 average temperature, were statistically relevant. These two variables influenced individually the model (Table 3 ). The predictions for 2007 per period of 3 months were improved after the introduction of either the minimal temperature at lag-5 weeks or the average temperature at lag-11 weeks (RMSE $=0.72$ and 0.74 , respectively, versus 0.85 for the univariate model) (Figures 5 and 6). However, these differences were not statistically significant (Wilcoxon signed-rank test: $\mathrm{p}$-value $=0.364$ and 0.407 , with lag-5 minimum temperature and lag-11 average temperature, respectively).

\section{Discussion}

We develop a model to improve the surveillance system and thus to help efficient dengue control. In the study, the predictions for the year 2007 per period of 3 months

Table 2 Characteristics of SARIMA models for climatic variables: coefficients, standard errors of residuals, AIC: Akaike information criterion, p-value after Ljung-Box test of residuals

\begin{tabular}{lllllccc}
\hline Climatic variables & SARIMA $(\mathbf{p}, \mathbf{d}, \mathbf{q})(\mathbf{P}, \mathbf{D}, \mathbf{Q})_{\mathbf{S}}$ model & AR1 & MA1 & SMA & Sd (residuals) & AIC & p-value (Ljung-box test) \\
\hline Accumulated rainfall & $(1,1,1)(0,1,1)_{52}$ & $0.154^{*}$ & $-0.980^{*}$ & $-1.00^{*}$ & 0.85 & 942.78 & $0.32(\mathrm{NS})$ \\
\hline Relative humidity & $(1,1,1)(0,0,0)_{52}$ & $0.207^{*}$ & $-0.632^{*}$ & - & 0.039 & -1320 & $0.307(\mathrm{NS})$ \\
\hline Minimum temperature & $(0,0,1)(0,1,1) 52$ & $0.249^{*}$ & - & $-1.00^{*}$ & 0.67 & 785.69 & $0.61(\mathrm{NS})$ \\
\hline Maximun temperature & $(1,1,1)(0,1,1)_{52}$ & $0.439^{*}$ & $-0.918^{*}$ & $-1.00^{*}$ & 0.473 & 576.63 & $0.566(\mathrm{NS})$ \\
\hline Average temperature & $(1,1,1)(0,1,1)_{52}$ & $0.310^{*}$ & $-0.927^{*}$ & $-0.897^{*}$ & 0.473 & 556.92 & $0.566(\mathrm{NS})$ \\
\hline
\end{tabular}

AR: autoregressive, MA: moving average, SMA: seasonal moving average, * :p-value $<0.05$ significant. 


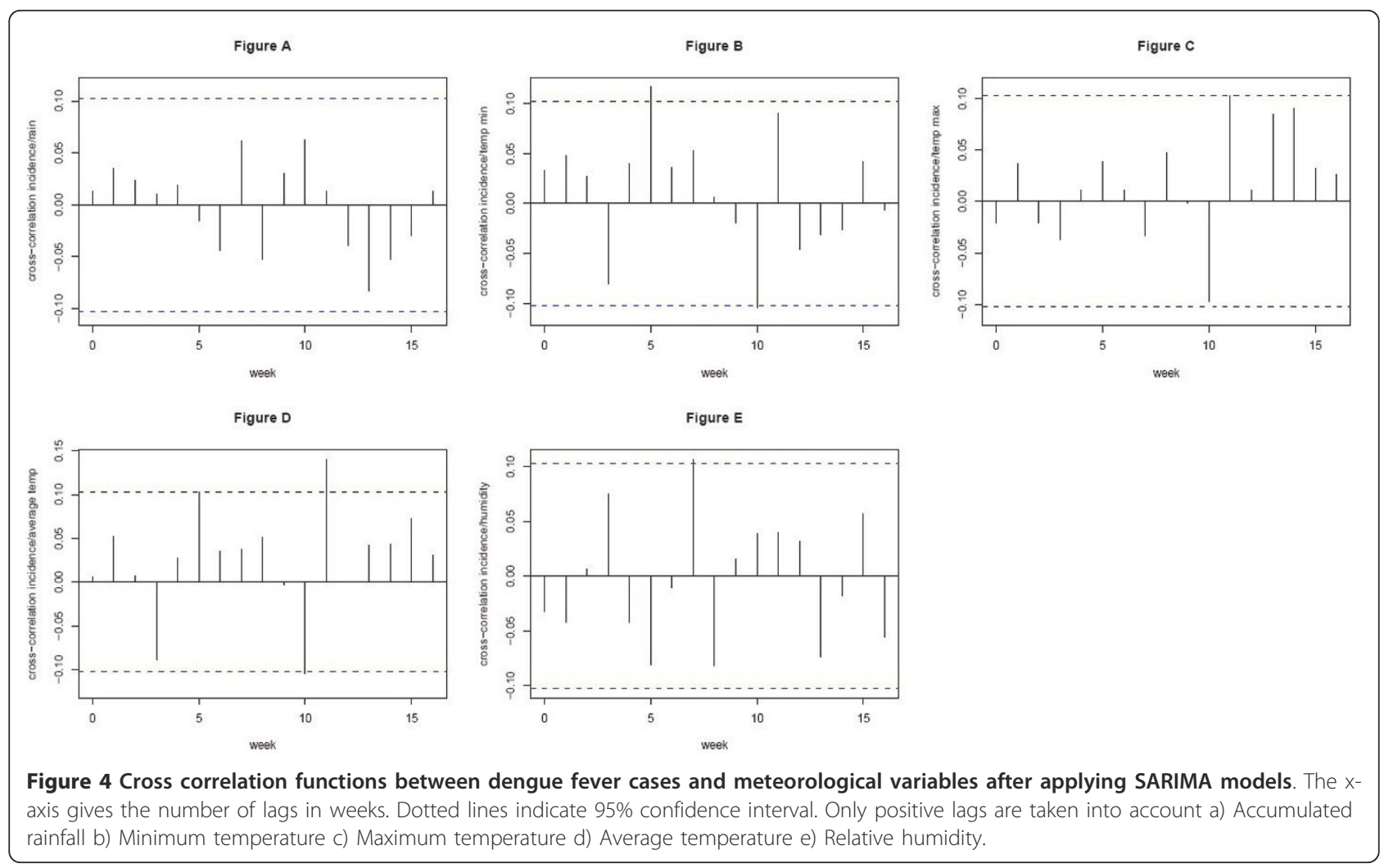

were improved after the introduction of the minimal temperature at lag-5 weeks or the average temperature at lag-11 weeks, but without reaching statistical significance when we compare univariate and multivariate SARIMA models. However, multivariate model can provide better prediction and might be implemented in routine for dengue surveillance in Guadeloupe.

This model, if tested in practice and validated with other set of data, might be useful for the evaluation of new intervention strategies introduced into this island.

The time series analysis has allowed the development of robust and reliable SARIMA models with a correct level of validity that fits dengue incidence data collected in Guadeloupe from 2000 to 2006 . It showed that the predictions over a period of 3 months for the year 2007 were the best way to implement appropriate prevention programs.
Moreover, the study demonstrated a positive correlation between dengue incidence and climatic variables such as relative humidity, minimum temperature and average temperature.

The predominant effect of these variables was observed after a 7 weeks-lag for the relative humidity, 5 weeks-lags for the minimum temperature, and 11 weeks-lags for the average temperature. Observed correlations are weak (below 0.2) since they are measured on residuals after an adjustment on the trend and the seasonality. However, they are statistically significant, which means the test is powerful, probably due to the high numbers of observations in the study.

These models present some limits. Firstly, the time series analysis requires homogeneous data over long periods, which are often difficult to obtain (i.e. the surveillance system in Guadeloupe was improved after 2004

Table 3 Characteristics of univariate and multivariate models using climatic variables the most correlated to dengue incidence: Coefficients, standard error and p-value of parameters, AIC, RMSE for predictions of the year 20073 months-ahead, * p-value $<0.05$ significant

\begin{tabular}{ccccccc}
\hline SARIMA model & Coefficients & Standard error & T- statistic & P-value & AIC & RMSE \\
\hline Univariate model & & & & & 951 & 0.85 \\
\hline Relative humidity_lag7 & 1.445 & 1.423 & 1.016 & 0.309 & 951 & 0.76 \\
\hline Minimum temperature _lag5 & $0.108^{*}$ & 0.068 & 1.593 & 0.030 & 950 & 0.72 \\
\hline Average temperature _lag11 & $0.228^{*}$ & 0.102 & 2.235 & 0.025 & 948 & 0.74 \\
\hline
\end{tabular}




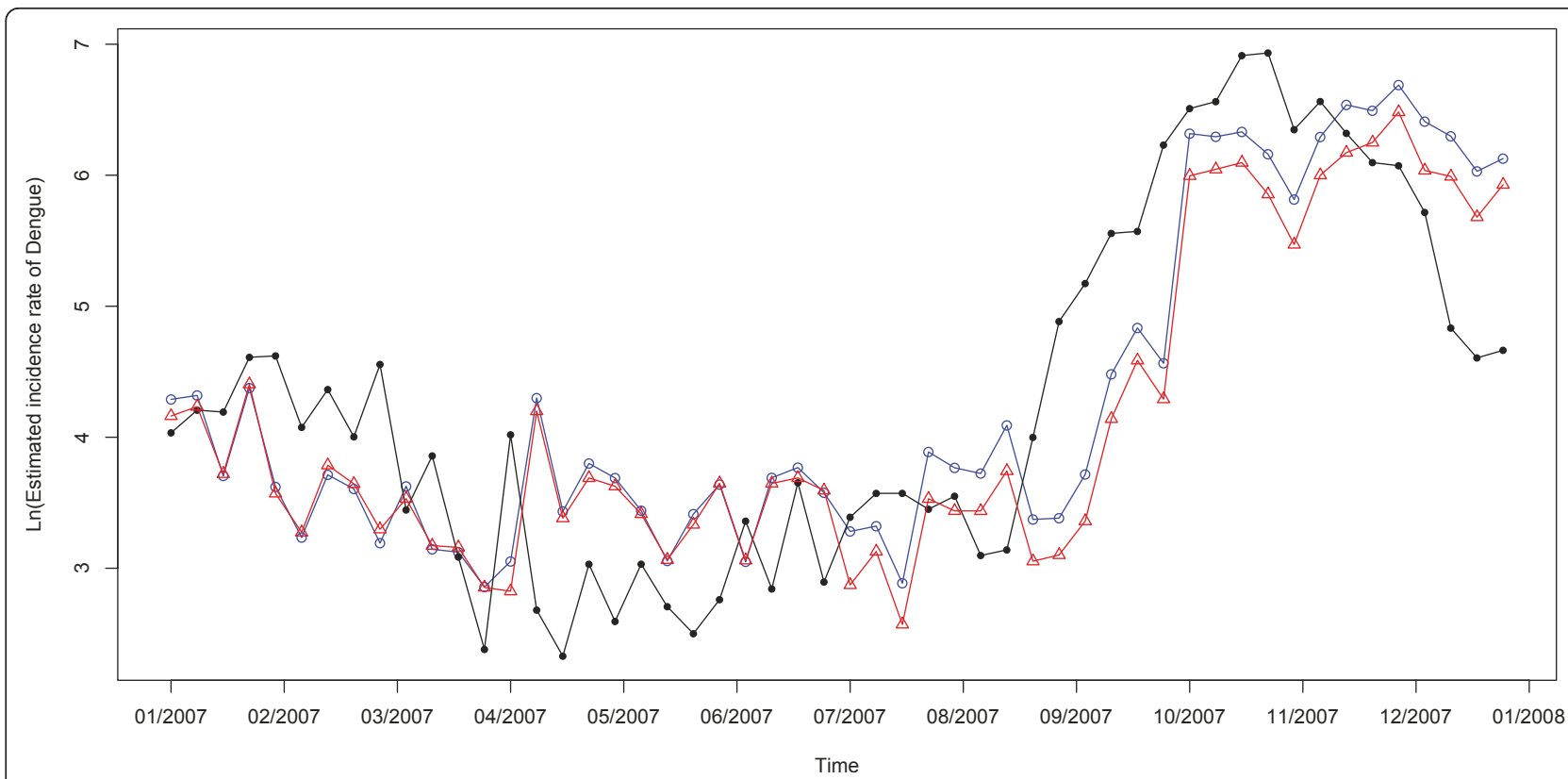

Figure 5 Natural logarithm of dengue incidence in Guadeloupe for 2007. Solid line (filled circle): observed values during the period. Red (triangle): univariate SARIMA $(0,1,1) \times(0,1,1) 52$ model's 13 weeks ahead values. Blue (circles): multivariate SARIMA $(0,1,1) \times(0,1,1) 52$ model's 13 weeks ahead values with minimum temperature lag-5.

providing heterogeneous data during the period of change). Ongoing dengue surveillance in Guadeloupe will allow local health authorities to improve this model since the reliability of predictions increases as time series become longer.
Secondly, the crude relationship between dengue incidence and climatic variables (Figure 1) shows that modification of the weather does not necessarily affect dengue epidemics while the use of SARIMA modelling, which is more relevant, points out a significant effect of

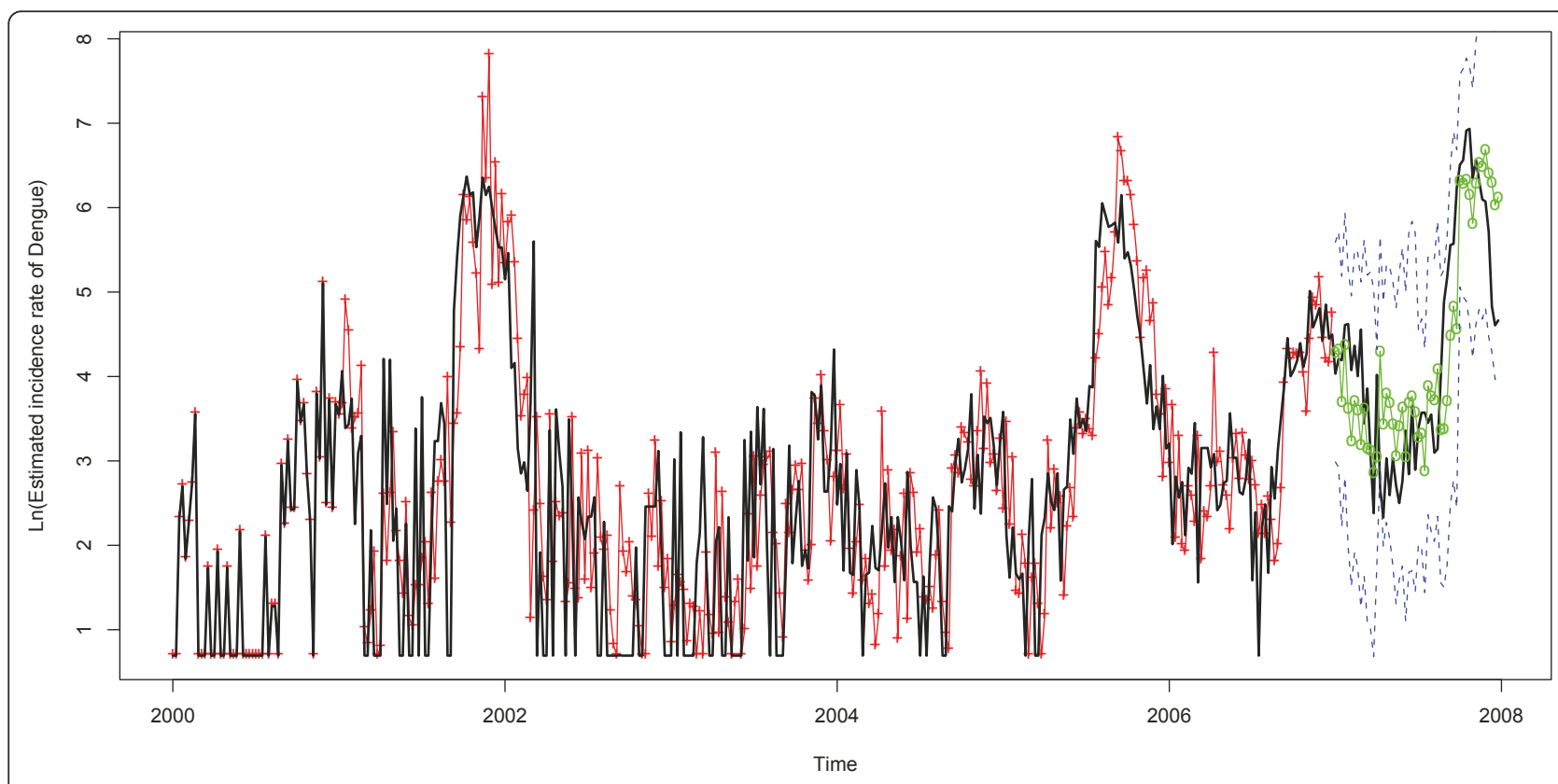

Figure 6 Natural logarithm of dengue incidence in Guadeloupe for the 2000-2007 period. Solid line: observed values during the period. Red (crosses): Fitted values from 2000 to 2006 and green (circles): predicted values for 2007 with multivariate SARIMA $(0,1,1) \times(0,1,1) 52$ model's 13 weeks ahead values with minimum temperature lag-5 as an external regressor and their $95 \%$ prediction intervals (Blue dashed lines). 
these variables. Some other climatic events not available for the study have not been analyzed: (1) vapor pressure which is a measure of relative humidity by satellite remote sensing [45] is less pertinent than humidity in our geographical situation because of the poor resolution in a small territory surrounded by water as the Guadeloupe Island; (2) the link between ENSO (El Niño-Southern Oscillation) and dengue incidence through mechanisms modifying the vector, the virus and the human behavior $[46,47]$ should be studied in the future to support our results. The delay between changes associated with ENSO and the increase in the dengue incidence could be as long as several months [46]; (3) the effect of hurricane in dengue virus circulation is not clear and difficult to take into account in the analyses. However, one of our teams (the Cire Antilles Guyane) tried to assess by a score the impact of the Hurricane "Dean" on the risk of dengue [48]. A deeper study should be done to evaluate correctly the effect of these strong events on the dengue incidence. Other non climatic factors might explain outbreaks of dengue such as (i) geographical characteristics of the study area (i.e. housing density, climate and agriculture works) and (ii) virus characteristics (serotype or virulence), but could not be explored in our study because of the non temporal format of these variables. However, the serotype should not affect so much the occurrence of epidemics because of the endemicity in the Caribbean region and the circulation of the four serotypes within a period of ten years. Most of the population has already been exposed to at least one serotype of dengue virus.

Finally, this model, which is global for Guadeloupe as a whole and not local by district, does not take into account geographical disparities in this island.

Our results are consistent with those of other studies dealing with the effect of climate on dengue outbreaks. These studies have highlighted that many climatic variables play a key role in dengue transmission. Although rainfall plays an ambiguous role on dengue incidence, temperature and relative humidity affect this transmission in several ways.

The potential impact of rainfall could either increase the transmission of vector-borne diseases, by promoting the proliferation of breeding sites, or eliminate breeding sites by flooding them $[18,49,50]$.

More than rainfall, temperature affects the dynamics of Ae aegypti by reducing the duration of the gonotrophic cycle [51], estimated at 6 to 7 days [52-54]. A proportional inverse effect is also observed in in vitro studies with duration range from 39.7 to 7.2 days for temperatures varying from $15^{\circ} \mathrm{C}$ to $35^{\circ} \mathrm{C}$ [55]. Moreover, temperature can increase the number of blood meals pro vector during each cycle amplifying significantly the level of transmission [56].
Relative humidity is another key factor that influences mosquitoes at different stages, especially during mating and egg laying. The combined effect of temperature and humidity influences significantly the number of blood meals and can also increase the survival rate of the vector, so that the probability it becomes infested during its life increases [57-59]. Moreover, higher temperatures make viral spread easier by reducing the extrinsic incubation period of Ae. aegypti (in vitro: 12 and 7 days, at $30^{\circ} \mathrm{C}$ and between $32^{\circ} \mathrm{C}$ and $35^{\circ} \mathrm{C}$, respectively [60]). In the literature, relative humidity and temperature are two important variables, more than rainfall. However, in Guadeloupe between 2000 and 2007, the relative humidity was a very stable parameter (mean $=79.3 \%$; IC95\% $[78.9,79.7])$. This very low range of variation might explain the low statistical power for this variable and the difficulty to point out a significant effect of the relative humidity in the SARIMA model.

Furthermore, the delayed effect of climatic variables on dengue incidence might be explained by climatic factors which do not influence directly the incidence of dengue but only indirectly through their effect on the life-cycle dynamics of both vector and virus. From mosquito hatching to human case appearance, several successive phases occur resulting in global cumulative lags observed in our study. These phases include larval and pupa development (10 to 21 days), gonotrophic cycle ( 3 to 7 days pro cycle), extrinsic incubation in mosquitoes (7 to 15 days), incubation in human (1 to 12 days). Depending on the respective lag between the biological cycle or mosquito life-stage and the clinical symptoms, the lag between weather data and incidence data will differ. The lag is expected to be shorter for minimum temperature that is usually associated with adult mosquito's mortality, longer for high relative humidity, both related to adult survival and hatching. On the other side, the mean temperature is involved in all biological cycles of Ae. aegypti that take more time to influence the dengue incidence.

Many studies have stressed a lag of several months. In Thailand, the dengue incidence was positively correlated with the average temperature at lag 3-4 months [19]. In Taiwan, there was a significant positive correlation with the maximum temperature at lag 1-4 months, the minimum temperature at lag 1-3 months and the relative humidity at lag 1-3 months [38]. In Brazil, positive associations were found between the minimum and maximum temperatures and dengue transmission at lag-0 [39]. And in the city of Guangzhou in China, the minimum temperature and relative humidity were positively correlated with the dengue incidence at lag-1 month [61].

Our results are consistent with all of these assumptions. We have shown that the increase in temperature 
and relative humidity were determining factors in predicting changes of the dengue incidence. On the contrary, rainfall did not appear to play a significant role. Actually, many breeding sites of Ae. aegypti are more dependent on human behavior than on rainfall for their constitution and persistence. That is the reason why local health authorities in Guadeloupe make people aware through sensitization campaigns since 2000. This might partly explain the lower impact of rainfall compared to other climatic variables on the dengue incidence.

We suggest that health authorities in other part of Caribbean or Latin America could introduce climatic variables in a local SARIMA approach to assess in another context the improvement of forecasting dengue incidence and outbreaks intensity.

The SARIMA modelling, used for predicting dengue incidence and outbreaks over a period of few months, must be considered as complementary from other modelling methods using non-epidemic data, as the Serfling regression model [62]. This model allows calculating a time-varying threshold, where an alert is generated if current data exceed the threshold. In Guadeloupe, such a modelling has been implemented since few years [63].

\section{Conclusion}

A reliable and robust model, that predicts the dengue incidence and outbreaks intensity over a period of 3 months, has been developed. This delay allows health authorities to launch an active vector control campaign, combining insecticide spraying and breeding sites destruction. Health facilities can also adapt patients care by increasing the number of available hospitalization beds if a dengue fever outbreak is predicted. This approach, which detects dengue epidemic risk and predicts outbreak intensity, fits very well with the current needs of the local health authorities. The model could contribute to dengue surveillance in Guadeloupe. The Cire Antilles-Guyane, department of public health in charge of surveillance data analysis, could update regularly the model, using data the most recently collected in the context of the ongoing and weekly dengue surveillance system. Therefore, they would obtain complementary information about outbreak risks.

\footnotetext{
Acknowledgements

Presented in part

11 èmes Journées Nationales d'Infectiologie, Montpellier, France, June 9-11, 2010

Congrès international d'épidémiologie "du nord au sud", Marseille, France, September 15-17, 2010

2e Journées Interrégionales de Veille Sanitaire des Antilles-Guyane, Cayenne, Guyane, October 8-9, 2010

We address special thanks to $\operatorname{Pr}$ A. Fontanet from Ecole Pasteur-Cnam de

Santé Publique in Paris who provides a general and substantial support in
}

this study and to Odile Faure from Department of Health and Social Development, Cellule de Veille Sanitaire for the collection of data. We thank Meteo France for providing meteorological data and the Fondation pour la Recherche Médicale for financial support to MG. The authors thank the Region Guadeloupe and the French Ministry of Overseas Territories for providing financial support.

\section{Author details}

${ }^{1}$ Ecole Pasteur-Cnam de Santé Publique, Paris, France. ${ }^{2}$ Regional office of the French Institute for Public Health Surveillance (Cire Antilles - Guyane), Fortde-France, Martinique, France. ${ }^{3}$ Department of Health and Social Development, Vector Control Department, Guadeloupe, France.

${ }^{4}$ International and Tropical Department, French Institute for Public Health Surveillance (InVS), Saint-Maurice, France. ${ }^{5}$ University of the French West Indies and of French Guyana, Guadeloupe, France. ${ }^{6}$ Research Unit "Epidemiology and Transmission of Emerging diseases", Institut Pasteur of Guadeloupe, Guadeloupe, France.

\section{Authors' contributions}

MG participated in the conception and the design of the study, performed the statistical analysis, participated in interpretation of data and in drafting the manuscript. PQ and SC participated in acquisition of data and revised the manuscript critically. JG has made substantial contributions to conception of the study. GLR participated in interpretation of data and revised the manuscript critically. LG helped to collect data. LM has proposed the research, made substantial contributions to conception and design of the study and coordination, participated in interpretation of data and has helped to draft the manuscript. All authors read and approved the final manuscript.

\section{Competing interests}

The authors declare that they have no competing interests.

Received: 8 October 2010 Accepted: 9 June 2011 Published: 9 June 2011

\section{References}

1. Lambrechts L, Scott TW, Gubler DJ: Consequences of the expanding global distribution of Aedes albopictus for dengue virus transmission. PLOS Negl Trop Dis 2010, 4:1-9.

2. World Health Organisation. Dengue: Guidelines for diagnosis, treatment, prevention and contro/ New Edition; 2009 [http://whqlibdoc.who.int/ publications/2009/9789241547871_eng.pdf].

3. Gubler DJ: Dengue/dengue haemorrhagic fever: history and current status. Novartis Found Symp 2006, 277:3-16.

4. World Health Organization: Asia-Pacific dengue program managers meeting Manila Western Pacific Region; 2008 [http://www.wpro.who.int/internet/ files/mvp/Dengue_Report.pdf].

5. Halstead SB: Dengue in the Americas and Southeast Asia: do they differ? Rev Panam Salud Publica 2006, 20:407-415.

6. Quénel P, Dussart P, Marrama L, Nacher M, Setbon M, Vergu E: Contributions de la recherche virologique, clinique, épidémiologique, socio comportementale et en modélisation mathématique au contrôle de la dengue dans les DFA. Bulletin de veille sanitaire 2009, 3:1-16[http://www.invs. sante.fr/publications/bvs/antilles_guyane/2009/bvs_ag_2009_03.pdf].

7. Wilson ME, Chen LH: Dengue in the Americas. Dengue Bull 2002, 26:44-61

8. Torres JR, Castro J: The Health and economic impact of dengue in Latin America. Cad Saude Publica 2007, 22(Suppl 1):S23-S31.

9. Cire Antilles-Guyane: Integrated management strategy for dengue prevention and control in the Caribbean subregion. Bulletin de veille sanitaire Antilles Guyane 2009, 8:2-15[http://www.invs.sante.fr/publications/ bvs/antilles_guyane/2009/bvs_ag_2009_08.pdf].

10. Gubler DJ: Dengue and dengue hemorrhagic fever. Clinical microbiology reviews 1998, 11:480-496.

11. Focks DA, Haile DG, Daniels E, Mount GA: Dynamic life table model for Aedes aegypti (Diptera: Culicidae): analysis of the literature and model development. J Med Entomol 1993, 30:1003-1017.

12. Focks DA, Daniels E, Haile DG, Keesling JE: A simulation model of the epidemiology of urban dengue fever: literature analysis, mode development, preliminary validation, and samples of simulation results. Am J Trop Med Hyg 1995, 53:489-506. 
13. Barbazan P, Yoksan S, Gonzalez JP: Dengue hemorrhagic fever epidemiology in Thailand: description and forecasting of epidemics. Microbes Infect 2002, 4:699-705.

14. Otero M, Solari HG: Stochastic eco-epidemiological model of dengue disease transmission by Aedes aegypti mosquito. Math Biosci 2010, 223:32-46.

15. Bartley LM, Donnelly CA, Garnett GP: The seasonal pattern of dengue in endemic areas: mathematical models of mechanisms. Trans $R$ Soc Trop Med Hyg 2002, 96:387-397.

16. Wearing HJ, Rohani P: Ecological and immunological determinants of dengue epidemics. Proc Natl Acad Sci USA 2006, 103:11802-11807.

17. Esteva $L$, Vargas $C$ : A model for dengue disease with variable human population. J Math Biol 1999, 38:220-240.

18. Gubler DJ, Reiter P, Ebi KL, Yap W, Nasci R, Patz JA: Climate variability and change in the United States: potential impacts on vector- and rodentborne diseases. Environ Health Perspect 2001, 109(Suppl 2):223-233.

19. Focks D, Alexander N, Villegas E: Multicountry study of Aedes aegypt pupal productivity survey methodology: Findings and recommendations. Denque bulletin WHO 2007, 31:192-200.

20. Nakhapakorn K, Tripathi NK: An information value based analysis of physical and climatic factors affecting dengue fever and dengue haemorrhagic fever incidence. Int J Health Geogr 2005, 4:13

21. Arcari P, Tapper N, Pfueller S: Regional variability in relationships between climate and dengue/DHF in Indonesia. Singapore Journal of Tropical Geography 2007, 28:251-272.

22. Bangs $M$, Larasati $R$, Corwin A, Wuryadi S, Jakarta I: Climatic factors associated with epidemic dengue in Palembang, Indonesia: implications of short-term meteorological events on virus transmission. Southeast Asian Journal of Tropical Medicine and Public Health 2006, 37:1103-1116.

23. Corwin A, Larasati R, Bangs M, Wuryadi S, Arjoso S, Sukri N, Listyaningsih E, Hartati S, Namursa R, Anwar Z: Epidemic dengue transmission in southern Sumatra, Indonesia. Transactions of the Royal Society of Tropical Medicine and Hygiene 2001, 95:257-265.

24. Burattini M, Chen M, Chow A, Coutinho F, Goh K, Lopez L, Ma S, Massad E: Modelling the control strategies against dengue in Singapore. Epidemiology and Infection 2007, 136:309-319.

25. Chowell G, Sanchez F: Climate-based descriptive models of dengue fever: the 2002 epidemic in Colima, Mexico. Journal of environmental health $2006,68: 40-44$

26. Keating J: An investigation into the cyclical incidence of dengue fever. Social Science \& Medicine 2001, 53:1587-1597.

27. Chen SC, Liao CM, Chio CP, Chou HH, You SH, Cheng YH: Lagged temperature effect with mosquito transmission potential explains dengue variability in southern Taiwan: Insights from a statistical analysis. Sci Total Environ 2010, 408:4069-4075.

28. Chadee D, Shivnauth B, Rawlins S, Chen A: Climate, mosquito indices and the epidemiology of dengue fever in Trinidad (2002-2004). Annals of tropical medicine and parasitology 2007, 101:69-77.

29. Barrera R, Delgado N, Jiménez M, Villalobos I, Romero I: Stratification of a city with hyperendemic dengue hemorrhagic fever. Revista Panamericana de Salud Pública 2000, 8:225-233.

30. Depradine C, Lovell E: Climatological variables and the incidence of Dengue fever in Barbados. Int J Environ Health Res 2004, 14:429-441.

31. World Health Organization: Using climate to predict Infectious Disease Outbreaks: A review Geneva; 2004 [http://www.who.int/globalchange/ publications/en/oeh0401.pdf]

32. Helfenstein $\mathrm{U}$ : The use of transfer function models, intervention analysis and related time series methods in epidemiology. Int J Epidemiol 1991, 20:808-815.

33. Choi K, Thacker SB: An evaluation of influenza mortality surveillance, 1962-1979. I. Time series forecasts of expected pneumonia and influenza deaths. Am J Epidemiol 1981, 113:215-226.

34. Allard R: Use of time-series analysis in infectious disease surveillance. Bull World Health Organ 1998, 76:327-333.

35. Nobre FF, Monteiro AB, Telles PR, Williamson GD: Dynamic linear model and SARIMA: a comparison of their forecasting performance in epidemiology. Stat Med 2001, 20:3051-3069.

36. Trottier H, Philippe P, Roy R: Stochastic modeling of empirical time series of childhood infectious diseases data before and after mass vaccination. Emerg Themes Epidemiol 2006, 3:9-24.

37. Quénel P, Dab W: Influenza A and B epidemic criteria based on timeseries analysis of surveillance data. European Journal of Epidemiology 1999, 14:275-285.
38. Wu PC, Guo HR, Lung SC, Lin CY, Su HJ: Weather as an effective predictor for occurrence of dengue fever in Taiwan. Acta Trop 2007, 103:50-57.

39. Luz PM, Mendes BV, Codeco CT, Struchiner CJ, Galvani AP: Time series analysis of dengue incidence in Rio de Janeiro, Brazil. Am J Trop Med Hyg 2008, 79:933-939.

40. Chaud P, Blateau A, Decludt B, Lamaury I, Strobel M, Talarmin A, Yebakima A: Guidelines for surveillance of dengue fever in the French Departments of America Martinique, Guadeloupe, Guyane; 1999 [http:// www.invs.sante.fr/publications/basag/basag2008_10.pdf].

41. Cassadou S: Le réseau de médecins sentinelles en Guadeloupe. Bulletin d'Alerte et de Surveillance Antilles Guyane 2008, 10:3-4[http://www.invs.sante $\mathrm{fr} /$ publications/basag/basag2008_10.pdf].

42. Box G, Jenkins G, Reinsel G: Time series analysis: forecasting and control San Francisco:Holden-day; 1976

43. Ljung G, Box G: On a measure of lack of fit in time series models. Biometrika 1978, 65:297-303.

44. Helfenstein U: Box-Jenkins modelling of some viral infectious diseases. Stat Med 1986, 5:37-47.

45. Hales S, de Wet N, Maindonald J, Woodward A: Potential effect of population and climate changes on global distribution of dengue fever: an empirical model. Lancet 2002, 360:830-834.

46. Hales S, Weinstein P, Souares $Y$, Woodward A: El Niño and the dynamics of vectorborne disease transmission. Environ Health Perspect 1999, 107:99-102.

47. Brunkard JM, Cifuentes E, Rothenberg SJ: Assessing the roles of temperature, precipitation, and ENSO in dengue re-emergence on the Texas-Mexico border region. Salud Publica 2008, 50:227-34.

48. CIRE Antilles-Guyane: Préparations épidémiologiques aux catastrophes naturelles. Bulletin d'Alerte et de Surveillance Antilles Guyane 2008, 9:1-11 [http://www.invs.sante.fr/publications/basag/Basag2008_9.pdf].

49. Woodruff RE, Guest CS, Garner MG, Becker N, Lindesay J, Carvan T, Ebi K Predicting Ross River virus epidemics from regional weather data. Epidemiology 2002, 13:384-393.

50. Kelly-Hope LA, Purdie DM, Kay BH: Ross River virus disease in Australia 1886-1998, with analysis of risk factors associated with outbreaks. J Med Entomol 2004, 41:133-150.

51. Scott TW, Morrison AC, Lorenz LH, Clark GG, Strickman D, Kittayapong P, Zhou H, Edman JD: Longitudinal studies of Aedes aegypti (Diptera: Culicidae) in Thailand and Puerto Rico: population dynamics. J Med Entomol 2000, 37:77-88.

52. Morrison A, Costero A, Edman J, Clark G, Scott T: Increased fecundity of Aedes aegypti fed human blood before release in a mark-recapture study in Puerto Rico. Journal of the American Mosquito Control Association 1999, 15:98-104.

53. Nayar J: Aedes aegypti (L.)(Diptera: Culicidae): Observations on dispersal, survival, insemination, ovarian development and oviposition characteristics of a Florida population. J Fla Anti-Mosquito Assoc 1981, 52:24-40.

54. Reiter P, Rodhain F: Oviposition et dispersion d'Aedes aegypti dans l'environnement urbain. Discussion. Bulletin de la Société de pathologie exotique 1996, 89:120-122.

55. Tun-Lin W, Burkot TR, Kay BH: Effects of temperature and larval diet on development rates and survival of the dengue vector Aedes aegypti in north Queensland, Australia. Med Vet Entomol 2000, 14:31-37.

56. Scott TW, Amerasinghe PH, Morrison AC, Lorenz LH, Clark GG, Strickman D Kittayapong P, Edman JD: Longitudinal studies of Aedes aegypti (Diptera: Culicidae) in Thailand and Puerto Rico: blood feeding frequency. J Med Entomol 2000, 37:89-101.

57. Rowley WA, Graham CL: The effect of temperature and relative humidity on the flight performance of female Aedes aegypti. J Insect Physiol 1968, 14:1251-1257.

58. McMichael A, Haines A, Slooff R, Kovats R: Climate change and human health: an assessment by a task group on behalf of the World Health Organization, the World Meteorological Organization and the United Nations Environment Programme Geneva: World Health Organization; 1996.

59. Thu HM, Aye KM, Thein S: The effect of temperature and humidity on dengue virus propagation in Aedes aegypti mosquitos. Southeast Asian $J$ Trop Med Public Health 1998, 29:280-284.

60. Watts DM, Burke DS, Harrison BA, Whitmire RE, Nisalak A: Effect of temperature on the vector efficiency of Aedes aegypti for dengue 2 virus. Am J Trop Med Hyg 1987, 36:143-152. 
61. Lu L, Lin H, Tian L, Yang W, Sun J, Liu Q: Time series analysis of dengue fever and weather in Guangzhou, China. BMC Public Health 2009,

9:395-400.

62. Serfling R: Methods for current statistical analysis of excess pneumoniainfluenza deaths. Public Health Reports 1963, 78:494-506.

63. Cire Antilles-Guyane: Elaboration d'un critère d'alerte pour la détection précoce des épidémies de dengue dans les Antilles françaises. Bulletin d'Alerte et de Surveillance Antilles Guyane 2008, 4:5-8[http://www.invs.sante.fr/ publications/basag/Basag2008-4.pdf].

\section{Pre-publication history}

The pre-publication history for this paper can be accessed here: http://www.biomedcentral.com/1471-2334/11/166/prepub

doi:10.1186/1471-2334-11-166

Cite this article as: Gharbi et al: Time series analysis of dengue incidence in Guadeloupe, French West Indies: Forecasting models using climate variables as predictors. BMC Infectious Diseases 2011 11:166.

\section{Submit your next manuscript to BioMed Central} and take full advantage of:

- Convenient online submission

- Thorough peer review

- No space constraints or color figure charges

- Immediate publication on acceptance

- Inclusion in PubMed, CAS, Scopus and Google Scholar

- Research which is freely available for redistribution

Submit your manuscript at www.biomedcentral.com/submit 